\title{
A Survey of Existing Mechanisms in Energy-Aware Routing In MANETs
}

\author{
Nazila Majdkhyavi \\ Departement of computer, \\ Meshkin branch, \\ Iclamic Azad University, Meshkin, Iran
}

\author{
Raziyeh Hassanpour \\ Departement of computer, \\ Urmia branch \\ Iclamic Azad University,Urmia, Iran
}

\begin{abstract}
A mobile ad hoc network (MANET) is a distributed and Self-organized network. In MANET, network topology frequently changes because of high mobility nodes. Mobility of nodes and battery energy depletion are two major factors that cause loss of the discovered routes. battery power depletion causes the nodes to die and loss of the obtained paths and thus affects the network connectivity. Therefore, a routing protocol for energy efficiency should consider all the aspects to manage the energy consumption in the network. so introducing an energy aware routing protocol, is one of the most important issues in MANET. This paper reviews some energy aware routing protocols. The main purpose energy aware protocols are efficiently use of energy, reducing energy consumption and increasing the network lifetime.
\end{abstract}

Keywords: MANET, routing, energy, transmission power, lifetime

\section{1-INTRODUCTION}

MANET is a collection of independent mobile nodes. In such a network, nodes can move freely and randomly. Network topology frequently changes and is unpredictable. This type of networks are limited in terms of resources such as power, memory and processing power [1].

Routing data packets in the network according to specific features of MANET, requires special mechanisms. In MANET, due to mobility of nodes and depletion of battery of nodes, the obtained paths are destroyed and data forwarding faces with failure and delay. In the event of any of these two events, the obtained routes will not be valid anymore and therefore the route discovery phase should be done again.

Re-runnig path discovery, imposes additional costs to the network, delays data forwarding and consumes network resources such as power and bandwidth. In the most of the existing routing protocols, there is a high probability to frequently select a node for data forwarding. This leads to high depletion rate of the node and cause the node to die and thus affects the network connectivity.

A routing protocol in ad hoc networks must be efficient in terms of energy consumption. This protocol must consider all aspects and factors of ad hoc networks to save energy. This is due to the nature of wireless networks which are battery constraint. Dynamic source routing (DSR) [2] and Ad hoc on demand distance vector (AODV) [3] routing protocols have been developed as the basic and most popular routing protocols by the Network Community for MANETs. However, these protocols have not been developed for energy efficient routing. Feeney [4] showed that these protocols are not optimized for energy efficient routing. Energy efficient routing have entirely different criteria than traditional metrics such as end to end delay and throughput and so on. So many researchers based on these protocols, have been proposed their own energy aware protocols.

Routing protocols in MANET are classified to reactive, proactive and hybrid ones. In this paper we focus on reactive routing protocols. The most famous of reactive protocols are DSR and AODV protocols. Most of the energy aware protocols consider these protocols as the underlying protocol to provide a new energy aware protocol. For example, the EDDSR protocol [8] is based on DSR and EA_AODV [17] and EEAODR [14] protocols are based on AODV. These protocols modified discovery/reply/maintenance phases of the traditional routing protocols to provide energy efficiency features.

The remainder of the paper is organized as follows. section 2 introduces some basic concepts about energy issues in MANET. section 3 introduces famous reactive protocols in MANET. Section 4 presents an overview of energy aware routing protocols in MANET and section 5 presents the conclusion of this paper.

\section{2-BASIC DEFINITIONS FOR ENERGY AWARE ROUTING}

In ad hoc networks, nodes are powered by batteries. Energy, communication and computational capacity at each node are limited. Nodes that lose all their energy, can not recharge their batteries and thus are removed from the network. For the below reasons, energy consumption management must be done:

-Limited energy storage: the nodes have limited battery power. 
-Battery replacement problems: in situations such as floods, earthquakes and battlefield, recharging or battery replacement is very difficult.

- lack of a central coordination: Due to the distributed nature of these networks and the lack of a central coordinator and multihop routing, some nodes act as relaying nodes and if the traffic load is heavy, it can consume a lot of energy of the relaying nodes.

-Battery resource constraints: node weight may increase by increasing the weight of the battery. By decreasing the weight of the battery, battery life may be reduced. So battery management techniques should be considered in these cases.

-choosing the optimal transmission power: with increasing the transmission power, the amount of battery consumption is also increases. Optimal transmission power reduces the interference between the nodes and therefore increases simultaneous transmissions.

High lifetime of the nodes can be achieved by increasing the capacity of the battery of nodes. Increasing the battery capacity of the nodes can be achieved with battery or power management.

\section{2-1 Energy Model}

Each node in the MANET consumes energy in four states:

- send

- Receive

- Idle

- Sleep

Suresh Singh, Mike Woo, and CS Raghavendra [5] described five energy aware criteria that affect the energy aware routing:

-minimizing the energy consumed per packet: This measures minimizes the average energy consumption per packet.

-Maximizing network partitioning time: The purpose of this measure is to increase the network lifetime.

-Minimizing difference in power levels of nodes: This criteria ensures that all the nodes in the network stay alive and work together.

-Minimizing cost per packet: This metric minimizes total cost of sending a packet.

-Minimizing the maximum cost of the node: This metric minimizes the maximum cost of the node.

\section{2-2 Energy Aware Routing}

Energy aware routing in MANET is of great importance. The main objective of energy aware routing protocols are reducing energy consumption in packet sending between source and destination, avoiding routing packets through nodes with low energy levels, optimizing the routing information in the network and increasing the network lifetime.

\section{3- REACTIVE ROUTING PROTOCOLS}

In reactive routing protocols for reducing overhead, the path between two nodes is established only when is needed. The most popular protocols in this area are DSR and AODV protocols. Most of the proposed energy aware protocols, have used DSR and AODV protocols as the underlying protocol. These protocols, with make some modification in route discovery/selection and in some cases route maintenance phase of the above mentioned protocols, have been added energy aware measures to these protocols.

\section{3-1 DSR Protocol}

In the DSR protocol, the source node generates a route request (RREQ) packet and inserts the source and destination address in the packet and broadcast it to all its neighbors. Each node receiving a RREQ packet, if did not know any path to the destination, inserts its own address into the path list of the packet and broadcast it. Upon recieving this packet on the destination, destination node creates a route reply (RREP) packet and sends it back to the source. When a node could not send a data packet to the next node on the path to the destination, generates a RERR packet and returns it to the source. The source node receiving RERR packet noticed a disconnection between the two nodes and route discovery phase is resumed.

\section{3-2 AODV Protocol}

In the AODV routing protocol, the routing operation is done hop by hop using routing tables. When a source wants to send data to a destination, if there is no route to the destination in its routing table, the RREQ message is broadcasted to all neighbors. Intermediate nodes can reply the RREQ packet if they have any path to the destination. To identify the fressness of the path provided by intermediate nodes, a sequence number are contained in the RREQ packets. When RREQ packet reaches the destination, destination node, sends a RREP packet back to the source using the reverse route. When traveling RREP packet on the reverse path, each intermediate node on the path establishes a forward path to the node from which the RREP packet is received.

\section{ENERGY AWARE ROUTING PROTOCOLS}

Energy aware routing in ad hoc networks is done in several ways. Some approaches try to minimize energy consumption during the route discovery/reply/maintenance. In this section some energy aware routing protocols for MANETs is presented. 


\subsection{Energy Aware Routing Protocols Based on DSR}

The DSR Protocol that is a source routing protocol, is one of the most popular protocols in MANETs. However, this protocol is not energy efficient. So many researchers with changes on this protocol phases and to improve its performance in terms of energy consumption, have added energy measures to the protocol. Some of these protocols are described below.

\section{4-1-1 Energy aware routing based on the energy drain rate}

In energy aware routing mechanisms based on drain rate (MDR) [6], a new cost function is presented to predict the lifetime of nodes. In this method, each node in the network includes its current cost in the received RREQ packet. During a certain interval $\mathrm{T}$ after receiving the first RREQ, the destination node, collects all the subsequent RREQs. When timer expires, the destination node chooses the routing path using Min-Max algorithm. Each node calculates its drain rate $\left(D_{\mathrm{i}}\right)$ every $\mathrm{T}$ seconds according to $\mathrm{DR}_{\text {old }}$ and $\mathrm{DR}_{\text {sample }}$ (old and new drain rate) values as relation (1):

$\mathrm{DR}_{\mathrm{i}}=\alpha \times \mathrm{DR}_{\text {old }}+(1-\alpha) \times \mathrm{DR}_{\text {sample }}$

In this protocol, the cost function $\mathrm{C}_{\mathrm{i}}=\mathrm{RBP}_{\mathrm{i}} / \mathrm{DR}_{\mathrm{i}}$ specifies the lifetime of node $i$ in which $\mathrm{RBP}_{\mathrm{i}}$ is the remaining battery power of node i. MDR mechanism chooses route with the highest lifetime among all the possible routes.

\subsubsection{Local Energy Aware Routing Protocol}

In the local energy aware routing protocol (LEAR-DSR) [7] which is based on DSR, if the remaining battery power of a node is higher than a certain threshold, the node will participate in the route discovery. Otherwise, the node drops RREQ packet and generates a new message, called drop route request (DRREQ) to inform other node about the droped RREQ. Thus, the RREQ packet will reach destination only when it has passed through nodes with higher energy than the specified threshold.

The path inserted in the RREP packets is the shortest path among all the paths that have sufficient energy. Each node has already removed a RREQ packet or has received a DRREQ packet, reduces amount of its battery threshold using a specific adjustment value and only if its residual energy is higher than the new threshold, will broadcast the RREQ packet.

\subsubsection{A Novel DSR-Based Energy Efficient Routing Protocol}

In the DSR-based energy efficient routing protocol (EDDSR) [8], each node based on its current level of battery power, calculates its willingness to participate in routing packets. EDDSR mechanism tries to discourage nodes that have low lifetime from participating in the route discovery and as a result increase node and network lifetime.

In this protocol, each node in the network periodically computes its remaining battery power (RBP) and if it has enough battery power, will participate in the network operations. When the remaining battery power of a node is below a certain threshold, the node delays broadcasting of the received RREQ. This operation is done by calculating a time period which is inversely proportional to its expected lifetime i.e. $C_{i}=R_{B P} / D_{i}$. In the route maintenance phase of EDDSR, when the energy level of the node reaches below a critical threshold, node sends a RERR packet to the source.

\section{4-1-4 Energy Aware Multi-Path Source Routing Protocol}

Energy aware multi-path source routing protocol (MEADSR) [9], is based on DSR protocol. In this protocol two path from source to the destination node are found and are stored in the routing table. The primary route selection in MEA-DSR protocol is done using residual energy of nodes on the path and the total transmission power required to transmit data. About selection of second route, the path with high disjointness ratio from the first path has high priority. A new field called min_bat_lev which stores the minimum residual energy of nodes on the path is added to the route table and RREQ packets.

In the MEA-DSR, only the destination node can responds to the RREQ packet. When an intermediate node which is located in the neighborhood of the source node, recieves the RREQ packet, inserts its remaining energy in the min_bat_lev field. Otherwise, each intermediate node compares its residual energy with the min_bat_lev value contained in the packet. If it is smaller, replaces min_bat_lev value by its remaining energy. After receiving first RREQ, the destination node waits for more RREQs. After that, the destination node, choose a primary route. After selecting the primaary route, the destination node immediately sends a route reply packet to the source node.

\section{4-1-5 Energy Aware Multi-Path Routing Protocol}

Energy aware multi-path routing protocol (EMRP) [10] which is derived from DSR protocol, applies some changes in the phases of DSR protocol. In the route reply phase of EMRP, each node on the path, inserts its current status such as the distance between the node $\mathrm{i}$ with the next hop, number of retransmission attempts related to the last successful transmission, current length of queue and its current energy in the RREP packet. Firstly, the source node calculates weight of all possible paths using relation (2):

$\mathrm{W}=\sum_{\mathrm{i}=1}^{\mathrm{n}}\left(\alpha \times \mathrm{W}_{\text {energy }}^{\mathrm{i}}+\beta \times \mathrm{W}_{\text {queue }}^{\mathrm{i}}\right)$

In relation (2), $W_{\text {energy }}^{\mathrm{i}}$ is the energy weight of node $\mathrm{i}$ and $\mathrm{W}_{\text {queue }}^{\mathrm{i}}$ is the queuing weight of node $i$. the source node after calculating the weights of all paths, sortes the routes in descending order based on $\mathrm{W}$ and selects the best $\mathrm{N}$ sets of paths as primary path for sending real-time data and the $\mathrm{N}$ next sets of 
paths as backup paths.

\section{4-1-6 Power Aware Source Routing Protocol}

In the Power Aware Source Routing Protocol (PSR) [11], during the route discovery procedure, the path $\pi$ at time $t$ is choosen so that the cost function (3) is minimized:

$$
C(\pi, t)=\sum_{i \in \pi} C_{i}(t) \quad \text { where } \quad C_{i}(t)=\rho_{i} \cdot\left(\frac{F_{i}}{R_{i}(t)}\right)^{\alpha}
$$

In relations (3), $\rho_{\mathrm{i}}$ is the Transmission power, $\mathrm{Fi}$ is the capacity of battery, $\mathrm{Ri}$ is the remaining battery of node $\mathrm{i}$ at time $\mathrm{t}$ and $\alpha$ is a weighting factor. In the PSR protocol, all nodes except the destination, calculate their link costs $(\mathrm{Cij})$ and insert it in RREQ packet. When an intermediate node receives a RREQ packet, it sets a timer $\mathrm{Tr}$ and holds the cost in the packet header as Min-Cost. If another RREQ packets reach, their costs are compared with the saved Min-Cost value. If new packets cost is less than saved Min-Cost, Min-Cost is changed to the new value and new RREQ packet are sent. Otherwise, the new RREQ is droped. In PSR, after receiving the first RREQ, destination node waits for a threshold $T_{r}$. When this timer expires, the destination node chooses the path with the least cost and responds to the source.

\section{4-1-7 Energy Aware and Reliable Routing Protocol}

In the Energy aware and reliable routing protocol (EARR) [12], each node depends on its residual energy and traffic requirements of higher layer decides whether to send RREQ packets or not. Only when its residual energy is higher than the required energy, will send RREQ packets. In this protocol, only when the intermediate nodes of the route have enough energy, the destination will receive the RREQ packet.

So only the valid paths are selected as candidate paths and this will avoid reconstruction of path because of lack of enough battery power and thus the life of the network will be increased. In this protocol, when intermediate nodes decide to send RREQ, insert their current energy in the packet. In the route cache of EARR, remaining energy also is stored. In this protocol if multiple candidate paths exist, the path with maximum battery capacity is selected for data transmission.

\section{4-1-8 Reliable Energy and Signal Strength Aware Routing Protocol}

The main objective of Reliable energy and signal strength aware routing protocol (SEA-DSR) [13] is to improve the reliability of the discovered path with taking into account both signal strength and remaining energy of nodes. This protocol reduces link failure and increases network lifetime by distributing traffic load. The benefits of this protocol are reduction of path failure, packet loss, route discovery and routing control overhead.
SEA-DSR protocol, adds an additional field called trust count (RELCOUNT) to the RREQ packet header of DSR protocol. After receiving first RREQ packet, the destination sets a timer and stores all RREQ in its route cache. After the timer, chooses the path with high reliability factor and sends a RREP packet for that. Reliability factor for the path is calculated using relation (4).

Reliability Factor $=\frac{\text { Reliability Count }}{\text { No.of Hops }}$

\section{4-2 Energy Aware Protocols Based on AODV Protocol}

AODV routing protocol is a reactive routing protocol which only holds the next hop for each destination in the routing table. In this section an overview of the multiple energy aware routing protocols based on AODV are performed. The protocols for improving the efficiency of AODV, added energy measures to the protocol.

\section{4-2-1 Energy Efficient Routing Protocol}

In the energy efficient routing protocol (EEAODR) [14], an optimization function based on time, the amount of energy of nodes and number of hops factors decides which path is the best path. In this protocol, if the path has one node with low energy level, the optimizer function will not choose it. if time is low, the route will be shorter and less energy will be consumed. but some nodes may die too quickly. Therefore optimizer function should do a trade off between time and network/node lifetime.

In this protocol when the destination receives first RREQ, waits for a while and collects all the subsequent RREQs. After this time, the destination uses the optimizer function to choose the best path and inserts it in the RREP packet. The destination also chooses some backup routes to avoid wasting energy and time for re-calculation of paths.

\section{4-2-2 Distributed Energy Efficient Routing Protocol}

Distributed energy efficient routing protocol (DEEAR) [15], obtains the necessary information for energy aware routing only using RREQ packet and requires only battery power level of the network. In DEEAR, intermediate nodes control rebroadcast time of RREQ packets and nodes with relatively high battery power broadcast the RREQ packets sooner. DEEAR protocol does a trade off between min-hop path and the path with balanced energy consumption.

To estimate the average power, average battery energy of nodes on the path (i.e. P) (source and intermediate nodes) and number of hops (i.e. N) fields are added to the RREQ packet. To select the optimal route, the source, adds the amount of its battery to the $\mathrm{P}$ field and broadcast the RREQ. Average remaining battery, $\mathrm{P}$ new, of the path is calculate as:

$\mathrm{P}_{\text {new }}=\left[\left(\mathrm{P}_{\text {old }} * \mathrm{~N}\right)+\mathrm{B}_{\mathrm{i}}\right] /(\mathrm{N}+1)$ 
In the relation (5), $B_{i}$ is remaining battery power of node $i$ and $\mathrm{P}_{\text {old }}$ is the amount of $\mathrm{P}$ field in the RREQ packet. With receiving a RREQ packet, node $\mathrm{i}$ calculates average remaining energy (i.e. $\mathrm{E}_{\text {new }}$ ) based on the prior average remaining energy of network (i.e. Eold) and $\mathrm{P}_{\text {old }}$ using relation (6):

$\mathrm{E}_{\text {new }}=\left[\left(1-\alpha * \mathrm{E}_{\text {old }}\right]+\left(\alpha * \mathrm{P}_{\text {old }}\right)\right.$

\section{4-2-3 Energy Based QoS Routing Protocol}

In energy-based QoS Routing Protocol (EQRP) [16], the problem of QoS routing is considered. According to this protocol, nodes based on their energy, decide how long delay the RREQ packet transmission. delay calculation is defined as:

$$
\mathrm{W}_{\mathrm{e}}=0, \mathrm{E}_{\mathrm{r}}>\mathrm{E}_{\max } \quad, \quad \mathrm{W}_{\mathrm{e}}=\frac{\mathrm{E}_{\mathrm{i}}}{\mathrm{E}_{\mathrm{r}}}
$$

In the relations (7), $E_{r}$ is current residual energy of node, $E_{i}$ is initial energy of node $I$ and $E_{\max }$ is the maximum energy for each node. The key idea is that EQRP choose the nodes on the path based on their residual energy. Due to the energy depletion of nodes, the goal of this protocol is to reduce route reconstruction.

\section{4-2-4 Integrated Energy Aware Routing Protocol}

In the integrated energy aware routing protocol (EA_AODV) [17], when each intermediate node receives the RREP packet, estimates its remaining battery power by using the energy available at the node and the required power to transmit a packet at the node and appened it in the RREP packet. Each node on the path, estimates its battery and only if it is less than the value contained in RREP packet, it will be replaced. If multiple paths reach the source, the path which its minimum battery energy is maximum is selected.

Available energy level and power level are taken into account when making routing decisions. In this protocol, source node chooses the path based on load balancing method to balance the battery depletion and applies transmission power control during data sending.

\section{4-2-5 Energy Aware Routing Protocol}

In the Energy Aware Routing Protocol (AODVEA) [18], routing is based on the minimum residual energy. Node with the minimum residual energy is marked and the path that has the largest minimum residual energy is selected. For this purpose, a new field called residual energy (Min-RE) is added to RREQ and RREP control packets.

This field, shows the node with the minimum residual energy. In this protocol, when the source receives the first valid
RREP packet, starts the communication. Once the source node receives the next RREP packet starts the main algorithm. Sends RREQ packets to all its neighbors. Gets various routes to destination. Compares parameters of routes with respect to the remaining energy level and the minimum number of hops and then chooses the right path to the destination.

\section{4-2-6 Dynamic Energy Aware Routing Protocol}

Dynamic energy aware routing protocol (EA-DYMO) [19], works based on the DYMO protocol. DYMO is a successor of AODV protocol. DYMO is an energy and load aware routing protocol that tries to provide a mechanism to select the optimal route based on the residual energy and traffic load of each node. In this protocol, route selection factor is calculated as the ratio of average energy to the average traffic load. The path which has maximum PS value is chosen for sending data. These two factors in relation (8) are combined to calculate the route selection factor:

$\mathrm{PS}_{\mathrm{sdi}}=\frac{\mathrm{EF}_{\mathrm{sdi}}}{\mathrm{LF}_{\mathrm{sdi}}}$

In the relation (8), $\mathrm{EF}_{\text {sdi }}$ and $\mathrm{LF}_{\text {sdi }}$ are the average power and average traffic load of path i from source $S$ to destination D.

\section{4-2-7 Reliable and Energy Efficient Routing Protocol}

Reliable and energy efficient routing protocol (E2R2P) [20], uses a new concept called backbone nodes, which are nodes with optimal signal strength. This project focuses on the concept of battery power and signal strength or required energy for the routing process. Backbone nodes helps in the construction phase and finding new routes. Each node has a routing table entry for the number of backbone nodes connected to it, its battery status and signal strength.

when any of the nodes on the communication path is damaged or move out of communication range, the backbone nodes can take care of routing process. Backbone nodes broadcast their signal strength and power status to neighboring nodes. In this protocol, selection process is based on power and signal strength factors. Although the path may be longer, but the chosen path is very stable and reliable to deliver the data packets.

\section{4-2-8 Reliable and Energy Aware Routing Protocol}

Reliable and energy aware routing protocol (TEA-AODV) [21], uses battery power and reliability value of each node for a reliable and trusted routing. Trust value of each node is determined based on various parameters such as length of the association, ratio of the number of packets sent successfully by neighbors to the total packets sent to that neighbor and the average time spent to response to a RREQ. 
In the route discovery phase, the source node broadcast RREQ packet containing the values of trust and reliability of source. Upon receiving RREQ packet, each intermediate node measures its reliability and adds it to RREQ packet. When two or more RREQs reaches the destination, destination finds the most reliable path using average reliability. The average reliability is obtained using relation (9):

Avgerage reliability $=($ Cumulative reliability/Number of hops) (9)

\section{4-2-9 Local Energy Aware Routing Protocol}

In the local energy aware routing protocol (LEAR-AODV) [22], each node based on its remaining battery power $\mathrm{E}_{\mathrm{r}}$, decides whether transmit the received RREQ packet or not. If the battery is more than a certain threshold, RREQ packet is sent, otherwise the packet is droped. If all nodes on the path have enough energy, RREQ packet will reach its destination. In the route maintenance phase if the battery is below a threshold, a RERR packet is sent to the sender node that this is done locally in the node sending the RERR packet. In this protocol, when a node removes a RREQ packet, it will broadcast a new message called ADJUST_Thr. Next nodes towards the destination find out a RREQ has been removed and adjust their threshold value.

\section{4-2-10 Lifetime Prediction Routing Protocol}

In the Lifetime prediction routing protocol (LPR-AODV) [22], the path with high lifetime is chosen. This protocol uses battery lifetime prediction. Each node estimates its battery lifetime based on the past activity. In the route discovery phase, all nodes except the destination and source calculate their estimated lifetime. An additional field called Min-lifetime is added to each route request packet which shows the minimum lifetime.

In the route selection phase, the source node chooses the path with maximum lifetime. In this protocol when the estimated lifetime reaches below a threshold, node sends a RERR packet to the source. This decision is only based on node residual energy and its depletion rate.

\section{4-2-11 Load Aware Routing Protocol}

Load Aware Routing Protocol (ELB-MRP) [23], uses collision window size and queue size to calculate load at the node and its single hop neighboring nodes. Each node except source and destination, collects information about the collision window size $(\mathrm{ACW})$, power factor $(\mathrm{EF})$ and queue factor $(\mathrm{QF})$ for itself and its single hop neighbors. The cost for node $\mathrm{i}$ is calculated using relation (10):

$\mathrm{C}(\mathrm{i})=\mathrm{ACW} * \mathrm{EF} * \mathrm{QF}$

(10)

To discover Paths, intermediate nodes add their traffic and energy information to the RREQ packets. Hello packets also collect information about $\mathrm{ACW}, \mathrm{EF}$ and $\mathrm{QF}$ values. Using the neighbor information obtained from hello packets, intermediate nodes calculates the cumulative cost using relation (11) and adds it to the RREQ packet. If a node $i$, has neighbors $\mathrm{j}$ and $\mathrm{k}$, the cumulative cost of CC(i) is obtained using the relation (11):

$\mathrm{CC}(\mathrm{i})=\mathrm{C}(\mathrm{i})+(\mathrm{C}(\mathrm{j})+\mathrm{C}(\mathrm{k}))$

When the first RREQ reaches the destination, destination waits for more RREQs. Then destination, selects two paths with least cost as primary and backup path.

\section{CONCLUSIONS}

In this paper, an overview of recently proposed routing protocols which conidered energy metric of nodes to improve routing operation is introduced. no routing protocol can not provide the best performance in all the different scenarios and the performance of a network depends on the condition and parameters of the network. parameters such as node mobility and QoS features are the most important factors in ad hoc networks. In this paper we focused on the characteristics of the nodes energy and reviewed the protocols that considered energy measure as the main parameter.

\section{REFERENCES}

1] Subir Kumar Sarkar, T G Basavaraju and C Puttamadappa, "Ad hoc mobile wireless networks, principles, protocols and applications".

[2] D.B. Johnson, D.A. Maltz, "Dynamic Source Routing in Ad Hoc Networks", Mobile Computing, T. Imielinski and H. Korth, Eds., Kulwer, 1996, pp. 152-81 protocol for mobile ad hoc networks (RDMAR)”, CCSR ,UK.

[3] C.E. Perkins, E.M. Royer, "Ad-Hoc On Demand Distance Vector Routing", Proceedings of the 2nd IEEE Workshop on Mobile Computing Systems and Application New Orleans, LA, February 1999, pp. 90-100.

[4] Laura Marie Feeney. An energy consumption model for performance analysis of routing protocols for mobile ad hoc networks. Mobile Networks and Applications, 6(3):239-249, 2001.

[5] S. Singh, M.Woo, and C. S. Raghavendra, "Power-aware with Routing in Mobile Ad Hoc Networks," Mobicom 1998, Dallas, TX, 1998.

[6] D.Kim, J.J.Garcia-Luna-Aceves, K.Obraczka, J.Carlos Cano, P.Manzoni, "Power Aware Routing Mechanism Based on the Energy Drain Rate Metric in Mobile Ad Hoc Networks," 11 th IEEE International Conference on Computer Communications and Networks (IC3N), 2002.

[7] K.Woo, C.Yu, H.Y.Youn, and B. Lee,”Non-Blocking, Localized RoutingAlgorithm for Balanced Energy Consumption in Ad Hoc Networks," International Symposium on Modeling, Analysis and Simulation of Computer and Telecommunication System, MASCOTS, 2001.

[8] Garcia, J.-E.; Kallel, A.; Kyamakya, K.; Jobmann, K.; Cano, J.-C.; Manzoni, P, "A novel DSR-based energyefficient routing algorithm for mobile ad-hoc networks", 58th IEEEVehicular Technology Conference, 2003. 
[9] S Chettibi, M Benmohamed, “A Multipath Energy-Aware On demand Source Routing Protocol for Mobile Ad-Hoc Networks", arxiv.org, 2009.

[10] Meng Li, Lin Zhang, Victor O. K. Li, Xiuming Shan, Yong Ren, "An Energy-Aware Multipath Routing Protocol for Mobile Ad Hoc Networks", ACM Sigcomm Asia, 2005.

[11] Morteza Maleki, Karthik Dantu, and Massoud Pedram, "Power-aware Source Routing Protocol for Mobile Ad Hoc Networks", ACM, August 12-14, 2002.

[12] Fang Xie, Lei Du, Yong Bai, and Lan Chen, "Energy Aware Reliable Routing Protocolfor Mobile Ad Hoc Networks", IEEE Communications Society subject matter experts for publication in the WCNC proceedings, 2007.

[13] P.Srinivasan \& K.Kamalakkannan, "Signal Strength And Energy Aware Reliable Route Discovery In MANET", International Journal of Communication Network Security ISSN: 2231 - 1882, Volume-1, Issue-4, 2012.

[14] Sanjay Kumar Dhurandher, Sudip Misra, “An EnergyEfficient On-Demand Routing Algorithm for Mobile Ad-Hoc Networks", 15th IEEE International Conference on Electronics, Circuits and Systems, 2008.

[15] radhika d.joshi, priti p.rege, "Distributed Energy Efficient Routing in Ad Hoc Networks", IEEE, Fourth International Conference onWireless Communication and Sensor Networks, 2008.

[16] Jin LIAN, “An Energy-based QoS Routing Protocol in mobile Ad Hoc Network", IEEE, Pacific-Asia Conference on Circuits, Communications and System, 2009.
[17] M. Tamilarasi, T.G. Palanivelu, Integrated EnergyAware Mechanism for MANETs using On-demand Routing, International Journal of Computer and Information Engineering 2:3 2008 .

[18] Radhika D.Joshi, Priti p.Rege, "Energy Aware Routing in Ad Hoc Networks", 6th WSEAS International Conference on CIRCUITS, SYSTEMS,ELECTRONICS,CONTROL \& SIGNAL PROCESSING, Cairo, Egypt, Dec 29-31, 2007.

[19] Sumit Kumar Singh, Shiva Prakash, Kapil Kumar, "Energy Aware Dynamic On-deman (EA-DYMO)", International Journal of Computer Applications (0975 - 8887), Volume 25- No.11, July 2011

[20] G.Madhukar Rao, M.Nadeem Baig, Md. Fareed Baba, k.kanthi kumar," Energy Efficient Reliable Routing Protocol for Mobile Ad hoc Networks", IEEE, 2011.

[21] M. Pushpalatha, Revathi Venkataraman, and T. Ramarao, "Trust Based Energy Aware Reliable Reactive Protocol in Mobile Ad Hoc Networks", World Academy of Science, Engineering and Technology 322009.

[22] S.-M. Senouci and G. Pujolle, "Energy Efficient Routing in Wireless Ad Hoc Networks", IEEE International Conference on Communications, 2004.

[23] Sujatha P Terdal, Dr. V.D.Mytri, Dr. A.Damodaram, “A Load Aware Routing Mechanism for Improving Energy Efficiency in Mobile Ad Hoc Networks", International Journal of Computer Applications, November 2010. 